\title{
Optoacoustic imaging with synthetic aperture focusing and coherence weighting
}

\author{
C.-K. Liao, M.-L. Li, and P.-C. Li \\ Department of Electrical Engineering, National Taiwan University, No. 1, Sec. 4, Roosevelt Road, Taipei, Taiwan
}

Received April 7, 2004

\begin{abstract}
Optoacoustic imaging takes advantage of high optical contrast and low acoustic scattering and has found several biomedical applications. In the common backward mode a laser beam illuminates the image object, and an acoustic transducer located on the same side as the laser beam detects the optoacoustic signal produced by thermoelastic effects. A cross-sectional image is formed by laterally scanning the laser beam and the transducer. Although the laser beam width is generally narrow to provide good lateral resolution, strong optical scattering in tissue broadens the optical illumination pattern and thus degrades the lateral resolution. To solve this problem, a combination of the synthetic aperture focusing technique with coherence weighting is proposed. This method synthesizes a large aperture by summing properly delayed signals received at different positions. The focusing quality is further improved by using the signal coherence as an image quality index. A phantom comprising hair threads in a $1 \%$ milk solution was imaged with an optoacoustic imaging system. The results show that the proposed technique improved lateral resolution by $400-800 \%$ and the signal-to-noise ratio by 7-23 dB over conventional techniques. (C) 2004 Optical Society of America

OCIS codes: $110.5100,110.5120,170.5120$.
\end{abstract}

Optoacoustic (OA) imaging has been used to image the optical properties of biological tissues noninvasively. OA signals can be induced with pulsed-light irradiation. When biological tissues absorb the light energy, thermoelastic expansion in the tissues generates ultrasonic waves. ${ }^{1}$ These ultrasonic waves are detected by an ultrasonic transducer, and the signals can be used to reconstruct the optical absorption distribution inside the image object. In biomedical applications, OA imaging takes advantage of high optical contrast and low acoustic scattering and hence represents a promising tool for breast tumor prediction, ${ }^{2}$ epidermal melanin measurement, ${ }^{3}$ and monitoring of oxygenation in blood vessels. ${ }^{4}$ Three modes of OA imaging have been introduced in the literature: the tomography mode, ${ }^{5}$ the forward mode, and the backward mode. ${ }^{1}$ In the latter two modes a cross-sectional image is formed by laterally scanning both the laser beam and the transducer. However, strong optical scattering in biological tissues broadens the optical illumination pattern, thus degrading the lateral resolution in OA imaging.

In this study the synthetic aperture focusing technique (SAFT) combined with coherence weighting is employed to improve the degraded lateral resolution. The backward mode is employed, and the OA probe comprises an optical fiber and a needle hydrophone. Figure 1 illustrates the concept of the SAFT. The OA probe is mechanically scanned to acquire a scan line at each scan position. Then the SAFT synthesizes a large aperture by properly delaying and summing the signals received at adjacent scan lines ${ }^{6}$ :

$$
\operatorname{RF}_{\mathrm{SAFT}}(t)=\sum_{i=0}^{N-1} \mathrm{RF}\left(i, t-\Delta t_{i}\right)
$$

where $\mathrm{RF}(i, t)$ is the received OA signal at the $i$ th position, $\Delta t_{i}$ is the time delay applied to the signal of scan line $i$, and $N$ denotes the total number of adjacent scan lines included in the SAFT summation. $\Delta t_{i}$ corresponds to the acoustic propagation time from the synthetic focal point to the OA probe at the $i$ th position. $\quad N$ is determined by the angular extent of the $\mathrm{OA}$ radiation pattern, which is the product of the optical illumination pattern and the hydrophone's directivity pattern. A larger extent (i.e., larger $N$ ) indicates that a bigger OA aperture can be synthesized. In addition to improving the lateral resolution, the SAFT can also increase the signal-to-noise ratio (SNR). Assuming uncorrelated, additive noise (e.g., thermal noise), the SNR improvement is equal to $10 \log _{10} N$.

In addition to the SAFT, the focusing quality is further improved by utilizing the signal coherence. The coherence of the delayed signals included in the SAFT sum is estimated by the coherence factor $(\mathrm{CF}),{ }^{7,8}$ which is defined as

$$
\mathrm{CF}(t)=\frac{\left|\sum_{i=0}^{N-1} \mathrm{RF}\left(i, t-\Delta t_{i}\right)\right|^{2}}{N \sum_{i=0}^{N-1}\left|\mathrm{RF}\left(i, t-\Delta t_{i}\right)\right|^{2}} .
$$

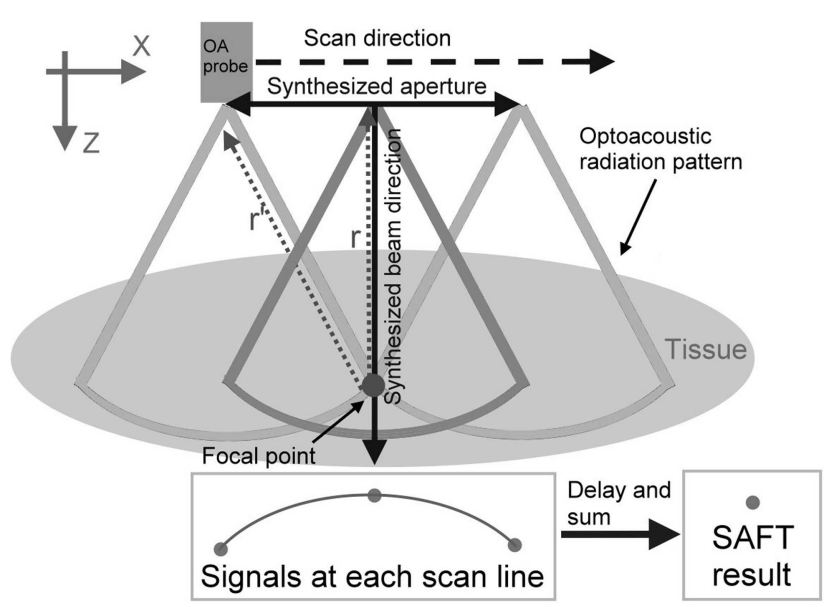

Fig. 1. Graphical illustration of the SAFT technique. 
The numerator in Eq. (2) represents the energy of the coherent SAFT sum, and the denominator denotes the total incoherent energy of the delayed signals included in the SAFT sum. According to the definition, the CF is a real quantity ranging from 0 to 1 . The $C F$ is maximal when the delayed signals are identical across the synthesized aperture (i.e., perfectly coherent), as illustrated in Fig. 2(a). Figure 2(a) shows the delayed scan lines corresponding to the case in which the point OA source is in the direction of the synthesized beam. In the figure the vertical axis is the depth and the horizontal axis is the synthesized aperture direction (i.e., the scan direction). In other words, the $\mathrm{CF}$ of an on-axis point source without focusing errors equals 1 . Figure 2(b) shows the case in which the point source is not on the synthesized beam axis, which corresponds to a steering error in the SAFT. The CF is low in this case since a destructive summation occurs in the numerator of Eq. (2). As illustrated in the above two examples, the $\mathrm{CF}$ can be used as a focusing-quality index for the SAFT. A high CF indicates that the image intensity should be maintained because the OA sources are in the direction of the synthesized beam. Otherwise, a low CF should be used to reduce the image intensity because of the presence of significant focusing errors. Based on this property, a coherencefactor-weighting (CFW) technique can be developed to further improve the focusing quality of the SAFT. The CF-weighted signal $\mathrm{RF}_{\text {weighted }}(t)$ of the SAFT signal $\operatorname{RF}_{\mathrm{SAFT}}(t)$ can be expressed as

$$
\mathrm{RF}_{\text {weighted }}(t)=\operatorname{RF}_{\mathrm{SAFT}}(t) \mathrm{CF}(t)
$$

Note that $\mathrm{CFW}$ has to be performed at each imaging point. Because measurement noise is generally incoherent, the $\mathrm{CFW}$ technique also improves the SNR. An OA scanning system has been built to test the efficacy of the SAFT and the CFW. This system consisted of a precision translation stage (CSR200, Control System in Motion), a laser system, and an acoustic-wave receiving system. A frequency-doubled Nd:YAG laser (LS-2132U, LOTIS TII) operating at $532 \mathrm{~nm}$ with a pulse duration of $8 \mathrm{~ns}$ was used for optical illumination. The laser beam was split and focused by lenses onto a 1000- $\mu \mathrm{m}$ fiber (FT-1.0-UMT, Thorlabs) with an optical energy of $1.67 \mathrm{~mJ}$ and a $15-\mathrm{Hz}$ pulse repetition rate. An ultrasonic needle hydrophone (GL-200, Onda) with a flat frequency response from 0.2 to $20 \mathrm{MHz}$ was employed to detect the OA waves. The active area of the hydrophone had a diameter of $200 \mu \mathrm{m}$. We constructed the OA probe by combining the fiber and the needle hydrophone on a single holder. This probe was mounted on the translation stage and mechanically scanned with a $100-\mu \mathrm{m}$ step size. The acoustic waveforms were amplified by an acoustic amplifier (5072PR, Panametrics) and then recorded by a data acquisition card (CompuScope 12100, Gage) at a $100-\mathrm{MHz}$ sampling rate. The acquired data were stored in a personal computer for subsequent data analysis.

The point-spread function of the imaging system was evaluated by considering a cross-sectional view of an OA hair-thread phantom. The OA phantom comprised six human black hairs $90 \mu \mathrm{m}$ in diameter, an $\sim 150-\mathrm{cm}^{-1}$ absorption coefficient, ${ }^{9}$ and a solution of $1 \%$ milk in water as the scattering medium with a scattering coefficient of $3.69 \mathrm{~cm}^{-1}$, which was measured by a double integrating sphere system. The hair threads were immersed in the solution and separated by $2 \mathrm{~mm}$ both axially and laterally, and they were the only absorbers in the phantom. The tip of the hydrophone was $3 \mathrm{~mm}$ below the surface of the solution and $5 \mathrm{~mm}$ above the first thread. Figure 3 shows the images of the OA phantom. Figures 3(a), $3(\mathrm{~b})$, and 3(c) are the original OA image, the SAFT image, and the SAFT-plus-CFW image, respectively. These images are displayed over a $30-\mathrm{dB}$ dynamic range. The vertical axis is the depth, and the horizontal axis is the lateral position, both in millimeters. Because of the broadened OA radiation pattern, the six hair threads shown in Fig. 3(a) have a wide lateral extent, indicating poor lateral resolution. The SAFT image in Fig. 3(b) exhibits improved lateral resolution and SNR: The former is due to the synthesis of a larger effective aperture, and the latter is a direct consequence of the delay and sum operations. The data from each synthesized aperture were also used

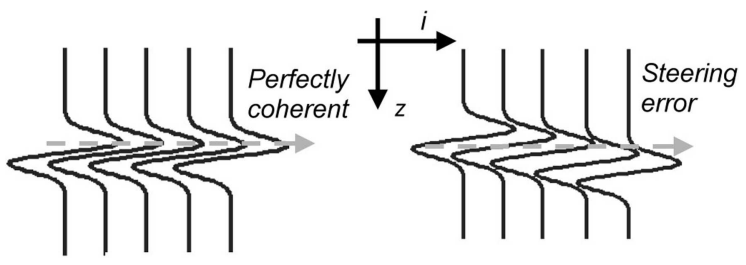

(a)

(b)

Fig. 2. Delayed scan lines. (a) Case in which the point OA source is in the direction of the synthesized beam without focusing errors. (b) Case in which the point OA source is not in the direction of the synthesized beam, corresponding to a steering error. The vertical axis is the depth, and the horizontal axis is the synthesized aperture direction.

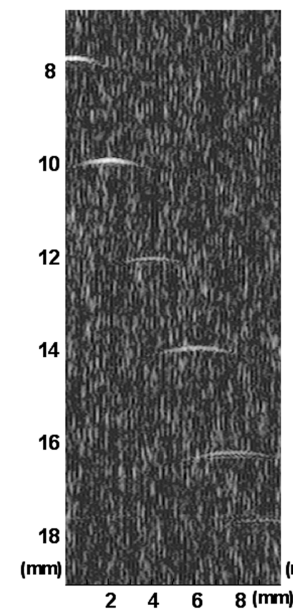

(a)

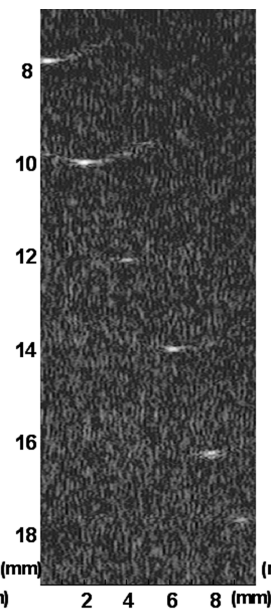

(b)

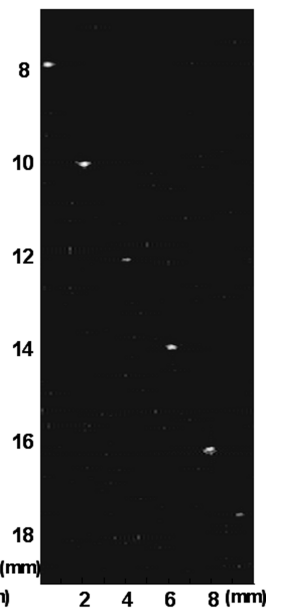

(c)
Fig. 3. Images of the $\mathrm{OA}$ phantom containing six hair threads: (a) original image, (b) SAFT image, (c) SAFTplus-CFW image. The dynamic range is $30 \mathrm{~dB}$. The vertical axis is the depth, and the horizontal axis is the lateral position, both in millimeters. 
Table 1. Lateral Width (at $-6 \mathrm{~dB}$ ) and SNR Values

\begin{tabular}{cccc}
\hline $\begin{array}{c}\text { Target Depth } \\
(\mathrm{mm})\end{array}$ & $\begin{array}{c}\text { Original } \\
(\mu \mathrm{m} / \mathrm{dB})\end{array}$ & $\begin{array}{c}\text { SAFT } \\
(\mu \mathrm{m} / \mathrm{dB})\end{array}$ & $\begin{array}{c}\text { SAFT }+ \text { CFW } \\
(\mu \mathrm{m} / \mathrm{dB})\end{array}$ \\
\hline 8 & $1200 / 25$ & $425 / 31$ & $250 / 41$ \\
10 & $1300 / 23$ & $550 / 32$ & $225 / 41$ \\
12 & $2700 / 13$ & $450 / 18$ & $280 / 26$ \\
14 & $1800 / 15$ & $400 / 27$ & $250 / 38$ \\
16 & $2000 / 16$ & $420 / 21$ & $260 / 33$ \\
18 & $>2500 / 12$ & $450 / 15$ & $280 / 19$ \\
\hline
\end{tabular}

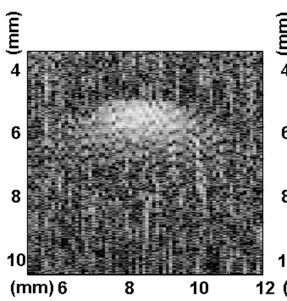

(a)

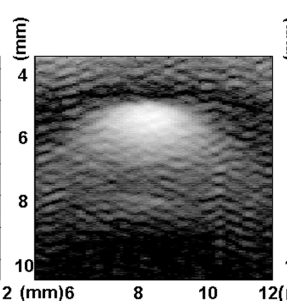

(b)

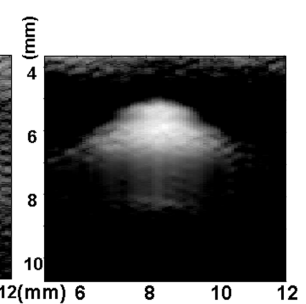

(c)
Fig. 4. Images of the cylindrical object: (a) original image, (b) SAFT image, (c) SAFT-plus-CFW image. The phantom consisted of poly(vinyl alcohol) ${ }^{10}$ gel and was located at a depth of $5 \mathrm{~mm}$. The diameter was $3.5 \mathrm{~mm}$ and had an absorption coefficient of $4.42 \mathrm{~cm}^{-1}$. The milk solution has a scattering coefficient of $3.69 \mathrm{~cm}^{-1}$.

to estimate the CF map. The final image produced by weighting (i.e., multiplication) of the SAFT image by the CF map on a pixel-by-pixel basis is presented in Fig. 3(c). Compared with the SAFT image shown in Fig. 3(b), the signals around the point source are noticeably suppressed. Moreover, the image background noise is reduced after the CFW.

The SNR and lateral resolution results for all targets are listed in Table 1, in which the SNR is defined as the difference between the maximal intensity at the hair-thread position and the average noise intensity. The wide OA radiation pattern led to broad widths of the images of the hair threads and the low SNR in the original OA image. The SAFT formed a narrow OA beam, which reduced the $-6-\mathrm{dB}$ width of the hair threads to $400-550 \mu \mathrm{m}$ at all depths. The lateral resolution was further improved with CFW to 225-280 $\mu \mathrm{m}$. Moreover, the SAFT improved the SNR by $3-12 \mathrm{~dB}$ and when combined with CFW provided an additional SNR improvement of 4-12 dB. The improved lateral resolution and SNR in the final image result in a reliable representation of this phantom. For further demonstration, images of a contrast phantom containing a poly(vinyl alcohol $)^{10}$ cylinder with a diameter of $3.5 \mathrm{~mm}$ are shown in Fig. 4. The cylinder was submerged at a depth of $5 \mathrm{~mm}$ in the milk solution (with the same scattering properties as the one shown in Fig. 3). Figure 4(a) shows the original image, Fig. 4(b) is the SAFT image, and Fig. 4(c) is the SAFT-plus-CFW image. Note that the upper edge of the cylinder was more visible than the lower edge. Such a characteristic is similar to that shown in Ref. 11. Both the edge definition and the SNR were markedly improved.

In summary, the SAFT-plus-CFW technique described here improves the degraded lateral resolution resulting from the strong scattering of light in OA imaging. The experimental results demonstrate the efficacy of the proposed technique: Both the SNR and the lateral resolution are improved. In general, the lateral resolution was improved by more than $400 \%$ and the SNR increased by $7-23 \mathrm{~dB}$. The next stage is to apply this technique in vivo.

The authors thank Deng-Huei Huang and Chen-Wei Wei for helpful comments and inspiring discussions related to this work. P.-C Li's e-mail address is paichi@cc.ee.ntu.edu.tw.

\section{References}

1. A. A. Karabutov, E. V. Savateeva, N. B. Podymova, and A. A. Oraevsky, J. Appl. Phys. 87, 2003 (2000).

2. R. O. Esenaliev, A. A. Karabutov, and A. A. Oraevsky, IEEE J. Sel. Top. Quantum Electron. 5, 981 (1999).

3. J. A. Viator, L. O. Svaasand, G. Aguilar, B. Choi, and J. S. Nelson, Proc. SPIE 4960, 14 (2003).

4. R. O. Esenaliev, I. V. Larina, K. V. Larin, D. J. Deyo, M. Motamedi, and D. S. Prough, Appl. Opt. 41, 4722 (2002).

5. X. Wang, Y. Pang, G. Ku, X. Xie, G. Stoica, and L. V. Wang, Nat. Biotechnol. 21, 803 (2003).

6. C. G. A. Hoelen and F. F. M. de Mul, Appl. Opt. 39, $5872(2000)$.

7. P.-C. Li and M.-L. Li, IEEE Trans. Ultrason. Ferroelectr. Freq. Control 50, 128 (2003).

8. M.-L. Li, W. J. Guan, and P.-C. Li, IEEE Trans. Ultrason. Ferroelectr. Freq. Control 51, 63 (2004).

9. "Significance of wavelength range for effective hair photo-epilation," (2004), http://www.depilazione.net/ news4.htm.

10. S. Manohar, A. Kharine, J. C. G. van Hespen, W. Steenbergen, F. F. M. de Mul, and T. G. van Leeuwen, Proc. SPIE 4960, 64 (2003).

11. J. J. Niederhauser, M. Jaeger, and M. Frenz, Proc. SPIE 4960, 118 (2003). 